\title{
Antimicrobial Activity of Mupirocin, Daptomycin, Linezolid, Quinupristin/ Dalfopristin and Tigecycline against Vancomycin-Resistant Enterococci (VRE) from Clinical Isolates in Korea (1998 and 2005)
}

\author{
Do Kyung Lee', Yuna Kim¹, Kun Sup Park ${ }^{1}$, Jae Wook Yang ${ }^{2}$, Kyungjae Kim ${ }^{1}$ and Nam Joo Ha ${ }^{1, *}$ \\ ${ }^{1}$ Department of Pharmacy, Sahmyook University, Seoul 139-742, Republic of Korea \\ ${ }^{2}$ School of Pharmacy, Western University, Pomona, California, USA
}

Received 23 March 2007, Accepted 21 June 2007

\begin{abstract}
It is a hot clinical issue whether newly approved antimicrobial agents such as daptomycin, linezolid, quinupristin/dalfopristin (synercid) and tigecycline are active enough to be used for infections caused by vancomycin resistant bacteria. We performed susceptibility tests for mupirocin, which is in widespread clinical use in Korea, and four new antimicrobials, daptomycin, linezolid, quinupristin/dalfopristin and tigecycline, against vancomycin-resistant Enterococcus faecalis and Enterococcus faecium isolated from Korean patients in 1998 and 2005 to evaluate and compare the in vitro activity of these antimicrobials. Among these agents, quinupristin/dalfopristin, which is rarely used in hospitals in Korea, showed relatively high resistance to several vancomycin-resistant enterococci (VRE) isolated in 2005. Likewise, daptomycin, linezolid and tigecycline have not yet been in clinical use in Korea. However, our results showed that most of the 2005 VRE isolates were already resistant to linezolid and daptomycin (highest minimum inhibitory concentration (MIC) value $>\mathbf{1 0 0} \mu \mathrm{g} / \mathrm{ml}$ ). Compared with the other four antimicrobial agents tested in this study, tigecycline generally showed the greatest activity against VRE. However, four strains of $\mathbf{2 0 0 5}$ isolates exhibited resistance against tigecycline (MIC $>12.5 \mu \mathrm{g} / \mathrm{ml}$ ). Almost all VRE were resistant to mupirocin, whereas all $E$. faecium isolated in 1998 were inhibited at concentrations between $0.8 \sim 1.6 \mu \mathrm{g} / \mathrm{ml}$. In conclusion, resistances to these new antimicrobial agents were exhibited in most of VRE strains even though these new antibiotics have been rarely used in Korean hospitals.
\end{abstract}

Keywords: Daptomycin, Linezolid, Tigecycline and vancomycin-resistant enterococci

\footnotetext{
*To whom correspondence should be addressed.

Tel: 82-2-3399-1607; Fax: 82-2-3399-1617

E-mail: hanj@syu.ac.kr
}

\section{Introduction}

Antimicrobial drug resistance has become a great public health problem and multidrug-resistant pathogens such as vancomycin-resistant enterococci (VRE) are on the increase worldwide in line with the increased consumption of glycopeptides (Centers for Disease Control and Prevention, 1993; Diekema and Jones, 2001; Kim et al., 2001; Jones, 2003).

VRE are of great concern because they are the most important causes of human nosocomial infections such as life threatening urinary tract infections, bacteremia, endocarditis, and others (Moellering, 1992; Emori and Gaynes, 1993; Klare et al., 1995; Witte, 1997; Patel, 2003). Moreover, VRE infections are difficult to treat, and few therapeutic options are currently available because these bacteria exhibit multidrug-resistance. Therefore, the spread of pathogens with this new antimicrobial drug resistance emphasizes the need for the development of other newer antimicrobial agents with activity against such pathogens (Wenzel, 2004; Fritsche et al., 2005; Nathwani, 2005).

Daptomycin, a cyclic lipopeptide produced by Streptomyces roseosporus, shows potent bactericidal activity against a wide spectrum of Gram-positive bacteria, including multidrugresistant strains, and may represent a reasonable therapeutic option for infections caused by these important pathogens. This antimicrobial has been recently approved by the United States Food and Drug Administration (FDA) for the treatment of complicated skin and soft tissue infections (Akins and Rybak, 2001; Sakouloas et al., 2003; LaPlante and Rybak, 2004; Sader et al., 2004; Streit et al., 2004; Wesson et al., 2004; Sader et al., 2005; Steenbergen et al., 2005).

Linezolid, the first approved oxazolidinone, has been utilized for infections caused by multidrug-resistant, Grampositive bacteria, especially VRE and methicillin-resistant staphylococci (Meka and Gold, 2004; Ross et al., 2004; Wesson et al., 2004). 
Quinupristin/dalfopristin (Synercid, 30:70 ratio) is the first parenteral streptogramin to have been recently licensed for clinical use in the United States and Europe for the treatment of infections caused by multidrug-resistant, Gram-positive pathogens (Raad et al., 2001; Raad et al., 2004; Wesson et al., 2004).

Tigecycline is a novel, broad-spectrum, parenteral glycylcycline that has been recently approved by FDA for treating infections of the skin and skin structures. It has been shown to be active against many Gram-positive, Gram-negative, atypical, and anaerobic organisms, including many resistant organisms such as penicillin-resistant Streptococcus pneumoniae (PRSP), methicillin-resistant Staphylococcus aureus (MRSA), and VRE (LaPlante and Rybak, 2004; Betriu et al., 2005; Bouchillon et al., 2005; Fritsche et al., 2005ab; Sader et al., 2005).

The aim of this study was to evaluate the in vitro activity of some newer antimicrobial agents that have not yet entered clinical use in Korea, against VRE isolated from Korean patients in 1998 and 2005.

\section{Materials and Methods}

Bacterial isolates. For this experiment, Enterococcus faecalis and Enterococcus faecium were obtained from C-hospital and Smedical laboratories in Korea in 1998 and 2005. The isolates were stored at $-70^{\circ} \mathrm{C}$ in brain heart infusion broth (Difco) supplemented with $20 \%$ glycerol before testing.

Antimicrobial agents. The following five antimicrobial agents were provided by their manufacturers for use in this study: mupirocin (Hanol), daptomycin (Cubist Pharmaceuticals) linezolid (Pharmacia), quinupristin/dalfopristin (Rhone-Poulenc Rorer) and tigecycline (Wyeth Pharmaceuticals)

Antimicrobial susceptibility test. The isolates were grown overnight on Mueller Hinton broth (Difco) at $37^{\circ} \mathrm{C}$ for $24 \mathrm{~h}$ and were tested for resistance to five antimicrobial agents such as mupirocin, daptomycin, linezolid, quinupristin/dalfopristin and tigecycline. MICs of the various antibiotics were determined by the agar dilution method according to the guidelines established by the National Committee for Clinical Laboratory Standards (NCCLS, 2003). For susceptibility of daptomycin, the test medium was Mueller Hinton agar adjusted to contain physiological levels of calcium (50 mg/L) as recommended by Fuchs et al., (2000).

MIC was defined as the lowest concentration of antimicrobial agent producing no visible growth of microorganism at $37^{\circ} \mathrm{C}$ after overnight incubation. Also, $\mathrm{MIC}_{50}$ and $\mathrm{MIC}_{90}$ was determined by the concentrations of antimicrobial agent at which $50 \%$ and $90 \%$ of microorganism are inhibited, respectively. The isolates were categorized as susceptible and resistant according to NCCLS guidelines (2003). The susceptible breakpoint for daptomycin and tigecycline was $\leq 4 \mu \mathrm{g} / \mathrm{ml}$ and susceptible breakpoint for enterococci was $\leq 0.5 \mu \mathrm{g} / \mathrm{ml}$, for which we followed criteria approve by the FDA (Package insert, 2003) and NCCLS (2005). The following quality control strains were concurrently tested by using $E$. faecalis ATCC 29212 and E. faecium ATCC 6057.

Genotypes (vanA and vanCl) of VRE were defined by PCRbased detection method as described previously (Choi et al., 2005; Acarturk et al., 2005; Yang et al., 2007).

\section{Results}

The results in Table 1 show increasing aspects of vancomycinresistant enterococci (VRE) in the Korean isolates of 2005 compared to 1998. Although $\mathrm{MIC}_{50}$ values of our isolates were same between 1998 and 2005, $\mathrm{MIC}_{90}$ value in isolates of 2005 were much higher than that of 1998. Several genes (vanA, vanB, vanCl,vanC2/3) associated with VRE phenotype have reported, and previous study by our study group detected the presence of vanA and vanCl genes in several strains among our isolates. VanA gene is observed in several Enterococcus spp. including E. faecalis and E. facium, and phenotypic expression by this gene occurs inducible resistance to both vancomycin and teicoplanin. In contrast, vanC gene is mainly observed in E. gallinarium, E. casseliflavus and E. flavescence, and characterized by constitutive low-level resistance to vancomycin but not to teicoplanin. Eight strains of the 1998 isolates had the vanCl gene, and showed lowlevel resistance to both vancomycin (MIC ranging from 0.8 $\mu \mathrm{g} / \mathrm{ml}$ to $6.25 \mu \mathrm{g} / \mathrm{ml}$ ) and teicoplanin (MIC ranging from 0.8 $\mu \mathrm{g} / \mathrm{ml}$ to $3.1 \mu \mathrm{g} / \mathrm{ml}$ ) (Table 2 and 4). Although usually detected among E. gallinarum (Choi et al., 2002), in this study the vanCl gene was found in many E. faecalis and E. faecium isolates. On the other hand, four strains among the 2005 isolates had the vanA gene (Yang et al., 2007) and showed high-level resistance to both vancomycin (MIC ranging 100

Table 1. Minimum inhibitory concentration(MIC) distributions for vancomycin of E. faecalis and E. faecium isolated from Korean patients in 1998 and 2005

\begin{tabular}{|c|c|c|c|c|c|c|c|c|c|c|c|c|c|c|}
\hline \multirow{2}{*}{ Antibiotic } & \multicolumn{2}{|c|}{ MIC $(\mu \mathrm{g} / \mathrm{ml})$} & \multicolumn{12}{|c|}{ Cumulative $\%$ inhibited at MIC $(\mu \mathrm{g} / \mathrm{ml})$} \\
\hline & $50 \%$ & $90 \%$ & 0.05 & 0.1 & 0.2 & 0.4 & 0.8 & 1.6 & 3.1 & 6.25 & 12.5 & 25 & 50 & 100 \\
\hline \multicolumn{15}{|l|}{1998 isolates } \\
\hline Vancomycin & 1.6 & 3.1 & 0 & 0 & 0 & 0 & 23 & 50 & 96 & 100 & - & - & - & - \\
\hline \multicolumn{15}{|l|}{2005 isolates } \\
\hline Vancomycin & 1.6 & $>100$ & 0 & 0 & 0 & 3 & 32 & 66 & 76 & 76 & 76 & 76 & 76 & 76 \\
\hline
\end{tabular}

MIC, minimum inhibitory concentration. 
Table 2. Minimum inhibitory concentration (MIC) of low level of vancomycin-resistant E. faecalis isolated from clinical isolates in Korea in 1998

\begin{tabular}{|c|c|c|c|c|c|c|c|}
\hline \multirow{2}{*}{ Strains/Year } & \multicolumn{7}{|c|}{$\mathrm{MIC}(\mu \mathrm{g} / \mathrm{ml})$} \\
\hline & VAN & TEI & MUP & DAP & ZYV & SYN & TIG \\
\hline \multicolumn{8}{|c|}{ Enterococcus faecalis } \\
\hline ATCC 29212 & 3.1 & 0.8 & 50 & 0.8 & 0.8 & 12.5 & 0.1 \\
\hline \multicolumn{8}{|c|}{ Enterococcus faecalis (1998) } \\
\hline Es $98-02$ & 3.1 & 1.6 & 50 & 3.1 & 0.8 & 6.25 & 3.1 \\
\hline Es $98-03$ & 1.6 & 0.8 & 50 & 0.8 & 0.8 & 6.25 & 3.1 \\
\hline Es 98-04 & 3.1 & 0.8 & 50 & 0.8 & 0.8 & 12.5 & 3.1 \\
\hline Es $98-05$ & 1.6 & 0.2 & 100 & 0.8 & 0.8 & 6.25 & 0.1 \\
\hline Es 98-06 & 3.1 & 1.6 & 50 & 1.6 & 0.4 & $>100$ & 1.6 \\
\hline Es $98-07$ & 3.1 & 0.8 & 50 & 1.6 & 0.8 & 25 & 3.1 \\
\hline Es $98-08$ & 1.6 & 0.8 & 50 & 0.8 & 0.4 & 25 & 1.6 \\
\hline Es 98-09 & 3.1 & 3.1 & 25 & 3.1 & 0.8 & 12.5 & 3.1 \\
\hline Es $98-10$ & 3.1 & 1.6 & 50 & 1.6 & 0.4 & $>100$ & 3.1 \\
\hline Es 98-11 & 1.6 & 0.8 & 50 & 0.8 & 0.8 & 6.25 & 3.1 \\
\hline Es 98-12 & 3.1 & 1.6 & 50 & 1.6 & 0.2 & $>100$ & 1.6 \\
\hline Es 98-13 & 3.1 & 0.8 & 50 & 1.6 & 0.8 & 6.25 & 3.1 \\
\hline Es 98-14 & 1.6 & 1.6 & 50 & 0.8 & 0.8 & 12.5 & 1.6 \\
\hline Es 98-15 & 3.1 & 0.8 & 50 & 0.8 & 0.8 & 12.5 & 3.1 \\
\hline Es 98-16 & 6.25 & 3.1 & 50 & 3.1 & 0.8 & 12.5 & 1.6 \\
\hline Es 98-17 & 3.1 & 0.8 & 50 & 0.8 & 0.8 & 12.5 & 3.1 \\
\hline Es 98-18 & 3.1 & 1.6 & 50 & 3.1 & 0.2 & $>100$ & 1.6 \\
\hline Es 98-19 & 3.1 & 1.6 & 50 & 3.1 & 0.8 & 12.5 & 1.6 \\
\hline
\end{tabular}

VAN; vancomycin, TEI; teicoplanin, MUP; mupirocin, DAP; daptomycin ZYV; linezolid, SYN; synercid (quinupristin/dalfopristin), TIG; tigecycline

$\mu \mathrm{g} / \mathrm{ml}$ ) and teicoplanin (MIC ranging from $6.25 \mu \mathrm{g} / \mathrm{ml}$ to 100 $\mu \mathrm{g} / \mathrm{ml}$ ) (Table 4).

Resistance to mupirocin was observed in almost all VREs, whereas all of E. faecium isolated in 1998 were inhibited at concentrations between $0.8 \sim 1.6 \mu \mathrm{g} / \mathrm{ml}$ (Table 4). For quinupristin/ dalfopristin, all $E$. faecalis including VRE were resistant, whereas for $E$. faecium, $74 \%$ and $100 \%$ of VSE (vancomycinsensitive enterococci) and VREs, respectively, exhibited resistance (Table 2, 3 and 4).

All 1998 isolates were susceptible to linezolid (Table 2 and 4), but $71 \%$ of VREs isolated in 2005 had developed resistance to this agent (Table 3 and 4).

Daptomycin was active against 1998 isolates. However resistance to this new agent was observed in most of VREs isolated in $2005\left(\mathrm{MIC}_{90}>100 \mu \mathrm{g} / \mathrm{ml}\right.$ ) (Table 3 and 4, Fig. 1 and 2). Resistance to daptomycin also tended to decrease with the increasing susceptiblity to vancomycin.

Tigecycline had greater activity against VRE than the other four antimicrobial agents in this study. All 1998 isolates and most of 2005 isolates, including VRE, were inhibited at concentrations between $<0.05 \sim 3.1 \mu \mathrm{g} / \mathrm{ml}$. However four strains of VRE isolated in 2005 exhibited a resistance against tigecycline (MIC $>12.5 \mu \mathrm{g} / \mathrm{ml}$ ) (Table 3 and 4). Tigecycline $\mathrm{MIC}_{90}$ level were $6.25 \mu \mathrm{g} / \mathrm{ml}$ and $12.5 \mu \mathrm{g} / \mathrm{ml}$ in vancomycin- resistant $E$. faecalis and vancomycin-resistant $E$. faecium isolated in 2005, respectively (Fig. 1 and 2). The highest tigycycline MIC value was $>100 \mu \mathrm{g} / \mathrm{ml}$ (Table 4). In particular, resistance to tigecycline was more frequent among 2005 isolates of $E$. faecium than of E. faecalis.

VRE exhibited multiple resistance and some of VREs were resistant to all new antimicrobial agents in this study.

\section{Discussion}

Since VRE was first isolated in Korea from nosocomial infection in 1992 (Kim and Song, 1998), the incidence of antimicrobial-resistant bacteria has continuously increased with the rising consumption of antibiotics. Especially, the development of resistance to glycopeptides such as vancomycin and teicoplanin has compromised medical treatment options because vancomycin remains the only alternative treatment for various life threatening infections in Korea, and many of the existing antimicrobials have become ineffective. Therefore, this decreased utility of many antibiotics has created a critical need for new therapeutic agents (Jones, 2003; Wenzel, 2004; Fritsche et al., 2005; Nathwani and Tigecycline, 2005).

In this study, we evaluated and compared new class 
Table 3. Minimum inhibitory concentration (MIC) of various levels of vancomycin-resistant $E$. faecalis isolated from clinical isolates in Korea in 2005

\begin{tabular}{|c|c|c|c|c|c|c|c|}
\hline \multirow{2}{*}{ Strains/Year } & \multicolumn{7}{|c|}{$\mathrm{MIC}(\mu \mathrm{g} / \mathrm{ml})$} \\
\hline & VAN & TEI & MUP & DAP & ZYV & SYN & TIG \\
\hline \multicolumn{8}{|c|}{ Enterococcus faecalis (2005) } \\
\hline Es $05-26$ & 1.6 & 0.4 & 25 & 0.8 & 0.8 & 12.5 & 1.6 \\
\hline Es $05-27$ & $>100$ & $>100$ & 100 & $>100$ & $>100$ & $>100$ & 1.6 \\
\hline Es $05-28$ & 1.6 & 0.4 & 12.5 & 0.8 & 0.8 & 12.5 & 0.2 \\
\hline Es $05-29$ & $>100$ & $>100$ & 100 & $>100$ & $>100$ & 100 & 6.25 \\
\hline Es $05-30$ & 0.8 & 0.8 & 0.8 & 3.1 & 0.8 & 12.5 & 0.1 \\
\hline Es $05-31$ & 1.6 & 0.2 & 12.5 & 0.8 & 0.8 & 25 & 0.1 \\
\hline Es $05-35$ & 3.1 & 0.8 & 25 & 1.6 & 0.2 & $>100$ & 1.6 \\
\hline Es $05-36$ & 1.6 & 0.2 & 25 & 0.8 & 0.8 & 12.5 & 0.1 \\
\hline Es $05-40$ & $>100$ & $>100$ & $>100$ & $>100$ & $>100$ & $>100$ & 6.25 \\
\hline Es $05-42$ & 1.6 & 0.2 & 25 & 3.1 & 0.8 & 12.5 & 0.1 \\
\hline Es $05-43$ & 1.6 & 0.1 & $<0.05$ & 0.8 & 0.8 & 25 & $<0.05$ \\
\hline Es $05-44$ & 1.6 & 0.2 & 25 & 1.6 & 0.8 & 6.25 & 0.1 \\
\hline Es $05-45$ & 1.6 & 0.2 & 25 & 0.8 & 0.8 & 12.5 & 0.1 \\
\hline Es $05-46$ & 1.6 & 0.2 & 25 & 0.8 & 0.8 & 25 & $<0.05$ \\
\hline Es $05-50$ & 1.6 & 0.4 & 25 & 0.8 & 0.8 & 6.25 & 0.2 \\
\hline Es $05-52$ & 1.6 & 0.4 & 25 & 0.8 & 0.8 & 12.5 & 0.1 \\
\hline Es $05-54$ & 1.6 & 0.4 & 50 & 1.6 & 0.8 & 12.5 & 0.1 \\
\hline Es 05-60 & 3.1 & $>100$ & $>100$ & 1.6 & 0.4 & $>100$ & 1.6 \\
\hline Es $05-62$ & 1.6 & 0.2 & 25 & 0.8 & 0.8 & 25 & 0.1 \\
\hline Es 05-63 & 1.6 & 0.4 & 25 & 0.8 & 0.8 & 12.5 & 0.1 \\
\hline Es 05-64 & $>100$ & 0.8 & 25 & $>100$ & $>100$ & 100 & 6.25 \\
\hline Es $05-67$ & $>100$ & $>100$ & $>100$ & $>100$ & $>100$ & $>100$ & 6.25 \\
\hline Es $05-65$ & 1.6 & 0.2 & 25 & 0.2 & 0.8 & 12.5 & 0.1 \\
\hline Es $05-71$ & 1.6 & 0.4 & 50 & 1.6 & 0.8 & 12.5 & 0.1 \\
\hline Es $05-72$ & 3.1 & $>100$ & $>100$ & 1.6 & $>100$ & $>100$ & 50 \\
\hline Es $05-78$ & 3.1 & 0.8 & $>100$ & 3.1 & 0.4 & $>100$ & 1.6 \\
\hline Es $05-79$ & 1.6 & 0.2 & 25 & 1.6 & 0.8 & 12.5 & 0.1 \\
\hline Es $05-83$ & 0.4 & 0.1 & 3.1 & 3.1 & 0.4 & 6.25 & 0.2 \\
\hline Es $05-86$ & 3.1 & 0.8 & 50 & 3.1 & 0.4 & 100 & 1.6 \\
\hline Es $05-92$ & 0.8 & 0.2 & 3.1 & 0.8 & 0.8 & 6.25 & 0.1 \\
\hline Es 05-94 & 1.6 & $<0.05$ & 50 & 1.6 & 0.8 & 25 & 0.1 \\
\hline
\end{tabular}

VAN; vancomycin, TEI; teicoplanin, MUP; mupirocin, DAP; daptomycin ZYV; linezolid, SYN; Synercid (quinupristin/dalfopristin), TIG; tigecycline

antimicrobial agents that possess activity for multidrugresistant bacteria. Recent studies (Hsueh et al., 2005; Smith et al., 2005) have shown newer agents, such as quinupristin/ dalfopristin, linezolid, daptomycin and tigecycline, have high activity against VRE and may therefore be therapeutic options for infections caused by these organisms. But the results of this study showed that excellent in vitro activity was not exhibited against VRE isolates as much as we expected.

Among these agents, quinupristin/dalfopristin (Synercid, $30: 70$ ratio) is the first parenteral streptogramin that has recently been licensed for clinical use in the United States and Europe for the treatment of infections caused by multidrugresistant, Gram-positive pathogens (Raad et al., 2001; Raad et al., 2004; Wesson et al., 2004). But this antibiotic is rarely used in Korean hospitals. However, in our study results, all $E$. faecalis and almost all VRE faecium were resistant to this agent. Several investigations have indicated that avoparcin used in animal husbandry has contributed to the increased pool of VRE present in animals, the environment and humans (Klare et al., 1995; Witte, 1997; WHO, 2001).

Likewise, it is suspected that the use of the streptogramin antibiotic virginiamycin as a feed additive in the commercial animal husbandry has contributed to the development of cross-resistance against streptogramin antibiotic (quinupristin/ dalfopristin). In addition, resistance to mupirocin was found in almost of $E$. faecalis including VRE. But all of $E$. faecium 
Table 4. Minimum inhibitory concentration (MIC) of various levels of vancomycin resistant $E$. faecium isolated from clinical isolates in Korea in 1998 and 2005

\begin{tabular}{|c|c|c|c|c|c|c|c|}
\hline \multirow{2}{*}{ Strains/Year } & \multicolumn{7}{|c|}{$\mathrm{MIC}(\mu \mathrm{g} / \mathrm{ml})$} \\
\hline & VAN & TEI & MUP & DAP & ZYV & SYN & TIG \\
\hline \multicolumn{8}{|c|}{ Enterococcus faecium } \\
\hline ATCC 6057 & 0.8 & 1.6 & 12.5 & 3.1 & 0.8 & 6.25 & 0.1 \\
\hline \multicolumn{8}{|c|}{ Enterococcus faecium (1998) } \\
\hline Em 98-02 & 0.8 & 3.1 & 0.8 & 6.25 & 0.8 & 6.25 & 0.1 \\
\hline Em 98-04 & 0.8 & 3.1 & 0.8 & 12.5 & 0.8 & 6.25 & 0.1 \\
\hline Em 98-05 & 1.6 & 3.1 & 0.8 & 6.25 & 0.8 & 1.6 & $<0.05$ \\
\hline Em 98-06 & 0.8 & 1.6 & 1.6 & 6.25 & 0.8 & 6.25 & 1.6 \\
\hline Em 98-07 & 0.8 & 3.1 & 1.6 & 12.5 & 0.8 & 3.1 & 0.1 \\
\hline Em 98-08 & 0.8 & 1.6 & 0.8 & 3.1 & 0.4 & 12.5 & 1.6 \\
\hline Em 98-14 & 0.8 & 1.6 & 0.8 & 3.1 & 0.4 & 25 & 1.6 \\
\hline Em 98-15 & 1.6 & 1.6 & 0.8 & 3.1 & 0.8 & 3.1 & 3.1 \\
\hline \multicolumn{8}{|c|}{ Enterococcus faecium (2005) } \\
\hline $\operatorname{Em} 05-16$ & $>100$ & $>100$ & 50 & $>100$ & $>100$ & $>100$ & 6.25 \\
\hline Em 05-17 & $>100$ & $>100$ & 50 & 6.25 & 100 & $>100$ & $>100$ \\
\hline Em 05-18 & $>100$ & $>100$ & $>100$ & $>100$ & 100 & $>100$ & 6.25 \\
\hline Em 05-19 & $>100$ & 12.5 & 0.8 & 3.1 & 0.4 & 6.25 & 1.6 \\
\hline$E m$ 05-20 & $>100$ & $>100$ & $>100$ & $>100$ & 100 & $>100$ & 12.5 \\
\hline Em $05-21$ & $>100$ & $>100$ & $>100$ & $>100$ & $>100$ & $>100$ & 12.5 \\
\hline Em $05-22$ & 0.8 & 0.8 & 25 & 3.1 & 0.4 & 12.5 & 1.6 \\
\hline Em $05-23$ & 0.8 & 0.4 & 0.2 & 1.6 & 0.8 & 1.6 & $<0.05$ \\
\hline Em 05-24 & $>100$ & 6.25 & 0.4 & 3.1 & 0.4 & 6.25 & 1.6 \\
\hline Em $05-25$ & 1.6 & 0.8 & 0.2 & 6.25 & 0.4 & 1.6 & $<0.05$ \\
\hline Em 05-26 & 1.6 & 0.8 & 0.8 & 6.25 & 0.8 & 3.1 & 0.1 \\
\hline Em 05-27 & 0.8 & 0.1 & 0.4 & 3.1 & 0.4 & 3.1 & 0.1 \\
\hline$E m$ 05-28 & 0.4 & 0.2 & 6.25 & 0.8 & 0.8 & 6.25 & 0.1 \\
\hline Em 05-29 & 0.8 & 0.8 & 0.4 & 6.25 & 0.8 & 3.1 & 0.1 \\
\hline Em $05-30$ & 0.8 & 0.8 & 0.4 & 6.25 & 0.8 & 50 & 0.1 \\
\hline$E m$ 05-31 & 0.8 & 0.4 & 3.1 & 6.25 & 0.4 & 12.5 & 0.1 \\
\hline Em 05-32 & 0.8 & 0.8 & 0.4 & 6.25 & 0.8 & 1.6 & $<0.05$ \\
\hline Em 05-33 & 0.8 & 0.8 & 0.4 & 6.25 & 0.8 & 3.1 & 0.1 \\
\hline Em 05-34 & 0.8 & 0.4 & 1.6 & 6.25 & 0.8 & 3.1 & 0.1 \\
\hline Em 05-35 & 3.1 & 0.8 & 25 & 1.6 & 0.4 & 100 & 1.6 \\
\hline Em 05-37 & 0.8 & 0.8 & 0.4 & 6.25 & 0.4 & 12.5 & 0.1 \\
\hline Em 05-38 & $>100$ & $>100$ & 0.8 & 3.1 & 0.4 & 3.1 & 1.6 \\
\hline$E m$ 05-39 & 0.8 & 0.8 & $>100$ & 6.25 & 0.4 & 3.1 & 0.1 \\
\hline Em $05-40$ & 0.8 & 0.8 & 0.8 & 6.25 & 0.4 & 3.1 & 0.1 \\
\hline$E m$ 05-41 & $>100$ & 25 & 0.4 & 1.6 & 0.4 & 12.5 & 1.6 \\
\hline Em 05-42 & 0.8 & 0.4 & 0.2 & 6.25 & 0.4 & 1.6 & 0.1 \\
\hline Em $05-44$ & 0.8 & 0.4 & 25 & 6.25 & 0.4 & 6.25 & 0.1 \\
\hline$E m$ 05-45 & 0.8 & 0.4 & 0.2 & 6.25 & 0.8 & 1.6 & 0.1 \\
\hline
\end{tabular}

VAN; vancomycin, TEI; teicoplanin, MUP; mupirocin, DAP; daptomycin ZYV; linezolid, SYN; Synercid (quinupristin/dalfopristin), TIG; tigecycline

isolated in 1998 were inhibited at concentration between $0.8 \sim$ $1.6 \mu \mathrm{g} / \mathrm{ml}$.

Linezolid is a new and unique antimicrobial class that has been introduced (oxazolidinones) and approved by the United States FDA in 2000 (Meka and Gold, 2004; Ross et al., 2004;
Wesson et al., 2004). This agent demonstrated good activity against all 1998 VRE isolates, but by 2005 most had developed resistance.

Daptomycin and tigecycline were approved in late 2003 and 2005, respectively, following their demonstration of 
Enterococcus faecalis

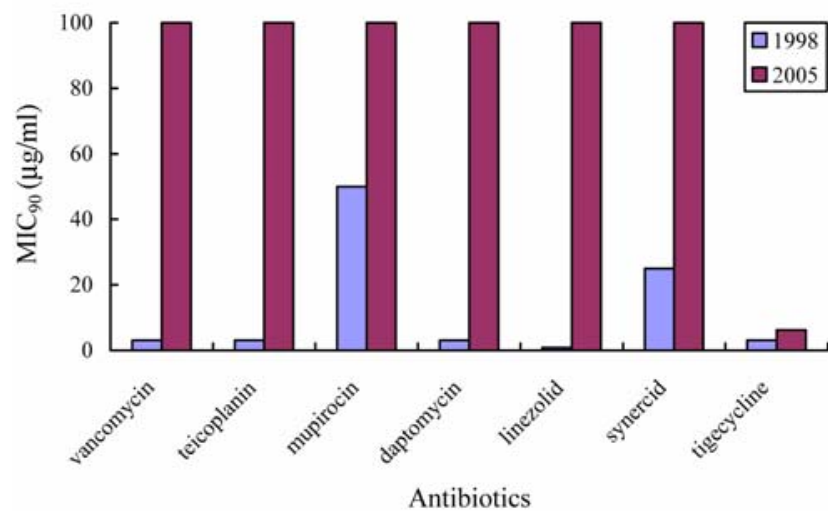

Fig. 1. $\mathrm{MIC}_{90}$ values for antibiotics of $E$. faecalis from clinical isolates in Korea in 1998 and 2005.

\section{Enterococcus faecium}

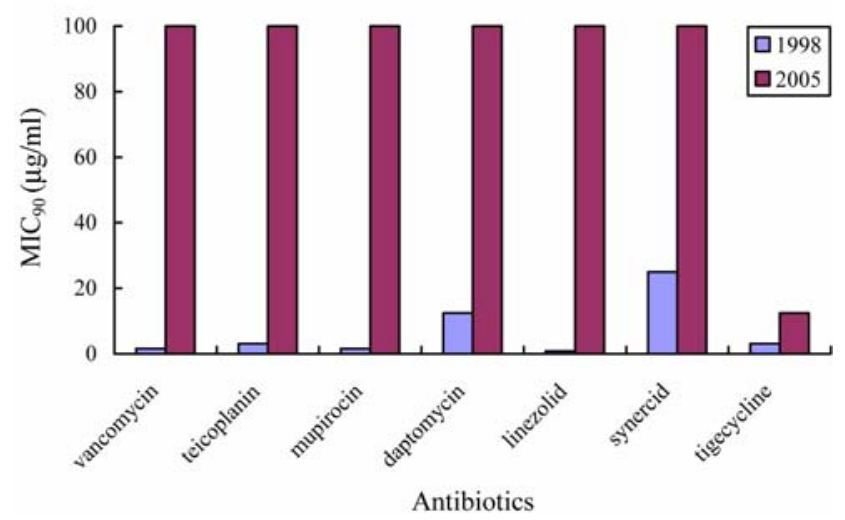

Fig. 2. $\mathrm{MIC}_{90}$ values for antibiotics of $E$. faecium isolated from clinical isolates in Korea in 1998 and 2005.

activity against various 1998 isolates including vancomycin and multidrug-resistant strains. Tigecycline was more highly active against VRE than the other four tested antimicrobial agents. However, the 2005 VRE isolates exhibited resistance to daptomycin and tigecycline. In particular, resistance to daptomycin tended to decrease with the increasing susceptibly to vancomycin. And a higher incidence of tigecycline resistance was observed among E. faecium than among $E$. faecalis in the 2005 isolates.

In conclusion, although newer antimicrobial agents such as daptomycin, linezolid, quinupristin/dalfopristin and tigecycline have not yet entered clinical use in Korea, some VREs isolated in 2005 have already begun to exhibit resistance to these agents. Although the new agents yet exhibited good activity against most of the isolates, they clearly do not promise to be long-term effective alternatives for various life threatening infections. In futute, the mechanism behind resistance against these new antimicrobial agents in some VREs should be clarified. Also, further clinical studies should consider the role that these agents may play in therapy for severe infections caused by VRE.

Acknowledgments This paper was supported by the Sahmyook University Research Fund in 2006. The authors are grateful to Sahmyook University and for the financial support provided by the Sahmyook University Research Fund.

\section{References}

Acarturk, E., Attila, G., Bozkurt, A., Akplnar, O., Matyar, S. and Seydaoglu, G. (2005) Insertion/deletion polymorphism of the angiotensin converting enzyme in coronary artery disease in Southern Turkey. J. Biochem. Mol. Biol. 38, 486-490.

Akins, R. L. and Rybak, M. J. (2001) Bactericidal activities of two daptomycin regimens against clinical strains of glycopeptide intermediate-resistant Staphylococcus aureus, vancomycinresistant Enterococcus faecium and methicillin-resistant Staphylococcus aureus isolates in and in vitro pharmacodynamic model with simulated endocardial vegetation. Antimicrob. Agents Chemother. 45, 454-459.

Betriu, C., Gomez, M., Rodriguez-Avial, I., Culebras, E. and Picazo, J. J. (2005) In vitro activity of tigecycline against ampicillinresistant Haemophilus influenzae isolates. J. Antimicrob. Chemother. 55, 809-810.

Bouchillon, S. K., Hoban, D. J., Johnson, B. M., Stevens, T. M., Dowzicky, M. J., Wu, D. H. and Bradford, P. A. (2005) In vitro evaluation of tigecycline and comparative agents in 3,049 clinical isolates: 2001 to 2002. Diagn. Microbiol. Infect. Dis. 51, 291-295.

Centers for Disease Control and Prevention (1993) Nosocomial enterococci resistant to vancomycin-United States, 1989-1993. Morbid. Mortal. Weekly Rep. 42, 597-599.

Choi, S. S., Kim, B. S. and Ha, N. J. (2002) Isolation, identification and characterization of vancomycin-resistant enterococci from raw milk. J. Microbiol. 40, 170-172.

Choi, J. H., Zhang, P. C., Park, K. W., Cho, Y. S. and Oh, B. H. (2005) The association between the T102C polymorphism of the HTR2A serotonin receptor gene and HDL cholesterol level in Koreans. J. Biochem. Mol. Biol. 38, 238-242.

Diekema, D. J. and Jones, R. N. (2001) Oxazolidinone antibiotics. Lancet 358, 1975-1982.

Emori, T. G. and Gaynes, R. P. (1993) An overview of nosocomial infections, including the role of microbiology laboratory. Clin. Microb. Drug Resist. 6, 313-318.

Fritsche, T. R., Sader, H. S., Stilwell, M. G., Dowzicky, M. J and Jones, R. N. (2005a) Antimicrobial activity of tigecycline tested against organisms causing community-acquired respiratory tract infection and nosocomial pneumonia. Diagn. Microbiol. Infect. Dis. 52, 187-193.

Fritsche, T. R., Sader, H. S., Stilwell, M. G., Dowzicky, M. J. and Jones, R. N. (2005b) Potency and spectrum of tigecycline tested against an international collection of bacterial pathogens associated with skin and soft tissue infections (2000-2004). Diagn. Microbiol. Infect. Dis. 52, 195-201.

Fuchs, P. C., Barry, A. L. and Brown, S. D. (2000) Daptomycin susceptibility tests: interpretive criteria, quality control, and effect of calcium on in vitro tests. Diagn. Microbiol. Infect. Dis. 38, 5158. 
Hsueh, P. R., Chen, W. H., Teng, L. J and Luh, K. T. (2005) Nosocomial infections due to methicillin-resistant Staphylococcus aureus and vancomycin-resistant enterococci at a university hospital in Taiwan from 1991 to 2003: resistance trends, antibiotic usage and in vitro activities of newer antimicrobial agents. Int. $J$. Antimicrob. Agents 26, 43-49.

Jones, R. N. (2003) Global epidemiology of antimicrobial resistance among community-acquired and nosocomial pathogens: a fiveyear summary from the Sentry Antimicrobial Surveillance Program (1997-2001). Semin. Respir. Crit. Care Med. 24, 121133.

Kim, J. M. and Song, Y. G. (1998) Vancomycin-resistant enterococcal infections in Korea. Yonsei Med. J. 39, 562-568.

Kim, S. M., Shim, E. S. and Seong, C. N. (2001) Prevalence and antibiotic susceptibility of vancomycin-resistant enterococci in chicken intestines and fecal samples from healthy young children and intensive care unit patients. J. Microbiol. 39, 116-120.

Klare, I., Heier, H., Claus, H., Reissbrodt, R. and Witte, W. (1995) VanA-mediated high-level glycopeptide resistance in Enterococcus faecium from animal husbandry. Fems Microbiol. Lett. 125, 165172.

LaPlante, K. L. and Rybak, M. J. (2004) Clinical glycopeptideintermediate staphylococci tested against arbekacin, daptomycin, and tigecycline. Diagn. Microbiol. Infect. Dis. 50, 125-130.

Meka, V. G. and Gold, H. S. (2004) Antimicrobial resistance to linezolid. Clin. Infect. Dis. 39, 1010-1015.

Moellering, R. C. (1992) Emergence of Enterococcus as a significant pathogen. Clin. Infect. Dis. 14, 1173-1178.

Nathwani, D. (2005) Tigecycline: clinical evidence and formulary positioning. Int. J. Antimicrob. Agents 25, 185-192.

National Committee for Clinical Laboratory Standards (NCCLS) (2003) Methods for dilution antimicrobial susceptibility tests for bacteria that grow aerobically. Approved standard M7-A6. Wayne PA: NCCLS.

National Committee for Clinical Laboratory Standards (NCCLS) (2005) Performance standards for antimicrobial susceptibility testing, $15^{\text {th }}$ information supplement M100-S15. Wayne PA: NCCLS.

Package insert (2003) Cubicin (daptomycin for infection). Lexington MA. (Cubist Phamaceuticals, Inc.) Available at http://www. cubist.com/shared/cubicin_label.pdf. Accessed on September 22, 2003.

Patel, R. (2003) Clinical impact of vancomycin-resistant enterococci. J. Antimicrob. Chemother. 51 (Suppl.), 13-21.

Raad, I., Hachem, R. and Hanna, H. (2001) Treatment of vancomycin-resistant enterococcal infections in the immunocompromised host: quinupristin-dalfopristin in combination with minocycline. Antimicrob. Agents Chemother. 45, 3202-3204.

Raad, I., Hachem, R., Hanna, H., Afif, C., Escalante, C., Kantarjian, H. and Rolston, K. (2004) Prospective, randomized study comparing quinupristin-dalfopristin with linezolid in the treatment of vancomycin-resistant Enterococcus faecium infections J. Antimicrob. Chemother. 53, 646-649.

Ross, J. E., Anderegg, T. R., Sader, H. S., Fritsche, T. R. and Jones, R. N. (2004) Trends in linezolid susceptibility patterns in 2002: report from the worldwide Zyvox Annual Appraisal of Potency and Spectrum Program. Diagn. Microbiol. Infect. Dis. 52, 53-58.

Sader, S. H., Fritsche, T. R. and Jones, R. N. (2005) Antimicrobial activity of daptomycin tested against clinical strains of indicated species isolated in North American medical centers (2003). Diagn. Microbiol. Infect. Dis. 53, 329-332.

Sader, H. S., Jones, R. N., Dowzicky, M. J. and Fritsche, T. R. (2005) Antimicrobial activity of tigecycline tested against nosocomial bacterial pathogens from patients hospitalized in the intensive care unit. Diagn. Microbiol. Infect. Dis. 52, 203-208.

Sader, H. S., Streit, J. M., Fritsche, T. R. and Jones, R. N. (2004) Antimicrobial activity of daptomycin against multidrug-resistant Gram-positive strains collected worldwide. Diagn. Microbiol. Infect. Dis. 50, 201-204.

Sakouloas, G., Eliopoulos, G. M., Alder, J. and Thauvin-Eliopoulos, C. (2003) Efficacy of daptomycin in experimental endocarditis due to methicillin-resistant Staphylococcus aureus. Antimicrob. Agents Chemother. 47, 1714-1718.

Smith, P. F., Booker, B. M., Ogundele, A. B. and Kelchin, P. (2005) Comparative in vitro activities of daptomycin, linezolid, and quinupristin/dalfopristin against Gram-positive bacterial isolates from a large cancer center. Diagn. Microbiol. Infect. Dis. 52, 255259.

Steenbergen, J. N., Alder, J., Thorne, G. M. and Talley, F. P. (2005) Dpatomycin: a lipopeptide antibiotic for the treatment of serious Gram-positive infections. J. Antimicrob. Chemother. 55, 283-288.

Streit, J. M., Jones, R. N. and Sader, H. S. (2004) Daptomycin activity and spectrum: A worldwide sample of 6,737 Grampositive organisms. J. Antimicrob. Chemother. 53, 669-674.

Wenzel, R. P. (2004) The antibiotic pipeline-challenges, costs and values. N. Engl. J. Med. 351, 523-526.

Wesson, K. M., Lerner, D. S., Silverberg, N. B. and Weinberg, J. M. (2004) Linezolid, quinupristin/dalfopristin, and daptomycin in dermatology. Dis. Mon. 50, 395-406.

Witte, W. (1997) Impact of antibiotic use in animal feeding on resistance of bacterial pathogens in humans. Ciba. Found. Symp. 207, 61-71.

World Health Organization (WHO) WHO Global strategy for containment of antimicrobial resistance. WHO/CDS/CSR/DRS/ 2001.2.

Yang, J. W., Lee, D. K., Kim, Y. A., Kang, B. Y., Kim, K. J. and Ha, N. J. (2007) Occurrence of the van genes in Enterococcus faecalis and Enterococcus faecium from clinical isolates in Korea. Arch. Pharm. Res. 30, 329-336. 